ORIGINAL ARTICLE

\title{
Preterm meconium staining of the amniotic fluid: associated findings and risk of adverse clinical outcome
}

\author{
A T Tybulewicz, S K Clegg, G J Fonfé, B J Stenson
}

Arch Dis Child Fetal Neonatal Ed 2004;89:F328-F330. doi: 10.1136/adc.2002.021949

See end of article for authors' affiliations

....................

Correspondence to:

Dr B Stenson, Neonatal

Unit, Simpson Centre for

Reproductive Health, Royal

Infirmary, Little France,

Edinburgh EH16 4SU,

Scotland, UK; ben.

stenson@luht.scot.nhs.uk

Accepted 17 July 2003
Background: The incidence of preterm meconium staining of the amniotic fluid (MSAF) is uncertain. It may be an indicator of possible listeriosis. It is unclear how great this risk is or whether preterm MSAF is a risk factor for adverse neonatal outcome.

Objective: To investigate the incidence of preterm MSAF, the incidence of associated maternal and neonatal infection, and the outcomes of the infants at discharge.

Design: Retrospective case-control study.

Methods: Infants $<33$ weeks gestation with preterm MSAF born in the Simpson Memorial Maternity Pavilion, Edinburgh between 1 January 1994 and 2 January 2001 were matched with the next infant of the same sex and gestation with clear liquor. Maternal and infant characteristics, culture results, placental histology, and clinical outcomes were compared.

Results: Preterm MSAF was observed in 45/1054 (4.3\%) infants below 33 weeks gestation. No maternal or infant listeriosis was identified in cases or controls. There was no significant difference in birth weight, Apgar score, or first pH between cases and controls. Preterm MSAF was associated with prolonged rupture of the membranes (odds ratio (OR) 3.34, 95\% confidence interval (CI) 1.07 to 10.49), but not maternal hypertension, sepsis, or chorioamnionitis. Severe (grade 3/4) intraventricular haemorrhage was significantly more common in infants with preterm MSAF (OR 2.03, 95\% Cl 1.62 to 2.53 ). There was no significant difference in mortality. Early onset sepsis was observed in two cases and three controls.

Conclusions: Preterm meconium staining of the amniotic fluid may be associated with increased risk of intraventricular haemorrhage. It does not appear to be a useful indicator of listeriosis.
$\mathrm{P}$ ublished data on meconium staining of the amniotic fluid (MSAF) in association with preterm delivery are limited. ${ }^{1-4}$ It is generally regarded to be uncommon, but the incidence is uncertain. It is unclear whether MSAF in preterm infants is a sign of illness or whether it predicts adverse outcome. MSAF has been noted in preterm infants with listeriosis. ${ }^{5}{ }^{6}$ Because of this association, preterm infants with MSAF often receive empirical treatment with ampicillin even though neonatal listeriosis is rare in the United Kingdom. The aims of this study were to investigate the incidence of preterm MSAF, to characterise the mothers and infants affected, and to determine the incidence of associated maternal and neonatal infection and the outcomes of the infants at discharge.

\section{METHODS}

A retrospective case-control study was performed. The study protocol was approved by the institutional ethics advisory committee. All infants born alive at the Simpson Memorial Maternity Pavilion in Edinburgh at less than 33 weeks gestation between 1 January 1994 and 2 January 2001 were included. Infants with MSAF observed clinically during delivery were identified from the case records. The infant and mother were matched with the next mother/infant pair of the same sex and gestational age with clear liquor. Because of the difficulty of identifying suitable controls, multiple pregnancies were matched to the next appropriate singleton mother/infant pair.

The maternal and infant case records were reviewed, and descriptive data, investigation results, clinical management decisions, and clinical outcomes were extracted. In all cases, the placenta was examined histologically by a specialist perinatal pathologist for signs of chorioamnionitis. Early onset neonatal sepsis was defined as present if a positive blood culture was obtained from the infant during the first 48 hours of life. Intraventricular haemorrhage was defined according to the Papile classification. Chronic lung disease was defined as a requirement for supplemental oxygen at 36 weeks gestational age.

Information on all cases of perinatal listeriosis identified in the institution during the study period was obtained from the Medical Microbiology and Pathology Departments.

\section{Statistical analysis}

Continuous data describing cases and controls were compared by Student's $t$ test, except Apgar scores, which were compared using the Mann-Whitney test. Differences in proportions were compared using the $\chi^{2}$ test. Odds ratios and $95 \%$ confidence intervals were calculated.

\section{RESULTS}

During the seven year study period, there were 39043 live births in the hospital, of which 1054 infants were less than 33 weeks gestation. MSAF was observed in 45/1054 of these infants $(4.3 \%)$. Table 1 gives the percentage of cases observed during each week of gestation. There were no cases at 23 or 24 weeks gestation. Other than this, there was no apparent effect of increasing gestational age on the incidence of MSAF in this population group.

Of the 45 case infants, seven were born of multiple pregnancies (one 28 week triplet pregnancy and two 30 week twin pregnancies). The 41 mothers of these case infants were matched to 41 control mothers with singleton pregnancies, yielding $4 \mathrm{l}$ case mothers, $4 \mathrm{l}$ control mothers, 45 case infants and 41 control infants.

Table 2 shows the maternal characteristics. Mothers of infants with preterm MSAF were more likely to have prolonged rupture of the membranes for $>24$ hours before 


\begin{tabular}{|llll|}
\hline $\begin{array}{l}\text { Table } 1 \\
\text { staining of the amniotic fluid (MSAF) by } \\
\text { gestation }\end{array}$ \\
\hline $\begin{array}{l}\text { Gestation } \\
\text { (weeks) }\end{array}$ & No of births & $\begin{array}{l}\text { No with } \\
\text { MSAF }\end{array}$ & $\begin{array}{l}\text { Percentage } \\
\text { with MSAF }\end{array}$ \\
\hline 23 & 28 & 0 & 0 \\
24 & 56 & 0 & 0 \\
25 & 43 & 2 & 4.7 \\
26 & 63 & 4 & 6.3 \\
27 & 52 & 3 & 5.8 \\
28 & 94 & 7 & 7.4 \\
29 & 141 & 7 & 5.0 \\
30 & 147 & 7 & 4.8 \\
31 & 186 & 7 & 3.8 \\
32 & 244 & 8 & 3.3 \\
All & 1054 & 45 & 4.3 \\
\hline \multicolumn{4}{r}{} \\
\hline
\end{tabular}

delivery, to be smokers, and to have concerns expressed in their delivery records about the cardiotocographic traces. Histological chorioamnionitis was equally common in cases and controls. There was no significant difference in the proportion of case and control mothers given antibiotics or steroids before delivery.

Table 3 shows the characteristics of the infants. Gestational age and sex were matched. There were no significant or clinically important differences in birth weight, Apgar scores, or first $\mathrm{pH}$ after birth between infants with preterm MSAF and controls. More case infants were intubated and more controls received surfactant, but these differences were not significant. Significantly more case infants were treated with antibiotics for more than 48 hours after birth than controls.

Table 4 shows the outcomes of the infants. Severe intraventricular haemorrhage (grade 3/4) was identified more often in infants with preterm MSAF than in controls (odds ratio 2.03, 95\% confidence intervals 1.62 to 2.53 ). Six infants in each group had grade $1 / 2$ intraventricular haemorrhage.

There was no difference in the proportions of infants with periventricular leucomalacia. More infants with preterm MSAF died or developed chronic lung disease than controls, but the differences were not significant. Of the seven infants born of multiple pregnancies, none died or developed severe intraventricular haemorrhage and one twin developed chronic lung disease.

Early onset sepsis was diagnosed in two case infants and three control infants: none of these infants died. None of the infants in either study group, or their mothers, had cultures positive for Listeria. There were three cases of perinatal listeriosis identified in the hospital during the study period. All had clear liquor. One of the three was stillborn at 26 weeks gestation. Two were live born at 32 and 34 weeks gestation respectively and both survived.

Table 2 Maternal characteristics

\begin{tabular}{lllr}
\hline & Cases & Controls & p Value \\
\hline Number & 41 & 41 & \\
Age & $28(7)$ & $28(6)$ & 0.73 \\
PROM & $24(59 \%)$ & $12(29 \%)$ & $<0.01$ \\
Chorioamnionitis & $10(24 \%)$ & $10(24 \%)$ & 0.95 \\
Antibiotics & $18(44 \%)$ & $13(32 \%)$ & 0.29 \\
CTG concern & $20(49 \%)$ & $10(24 \%)$ & 0.03 \\
Hypertension & $3(7 \%)$ & $7(17 \%)$ & 0.18 \\
Steroids & $37(90 \%)$ & $34(83 \%)$ & 0.78 \\
Smoker & $13(32 \%)$ & $5(12 \%)$ & 0.03 \\
\hline
\end{tabular}

Data are mean (SD) or number (\%).

PROM, Premature rupture of the membranes; CTG, cardiotocographic.
Table 3 Infant characteristics

\begin{tabular}{llll}
\hline & Cases & Controls & p Value \\
\hline Number & 45 & 41 & \\
Gestational age (weeks) & $28.7(4.6)$ & $28.9(2.2)$ & \\
Sex (M:F) & $19: 26$ & $18: 23$ & \\
Birth weight (g) & $1249(429)$ & $1241(401)$ & 0.93 \\
Apgar at 5 min & $8(5-10)$ & $9(7-9)$ & 0.79 \\
First pH & $7.32(0.09)$ & $7.3(0.14)$ & 0.88 \\
Intubated & $40(89 \%)$ & $32(78 \%)$ & 0.6 \\
Surfactant & $18(40 \%)$ & $24(59 \%)$ & 0.09 \\
Antibiotics $>48$ hours & $29(64 \%)$ & $13(32 \%)$ & 0.003 \\
\hline \multirow{2}{*}{ Data are mean (SD), number (\%), or median (interquartile range). }
\end{tabular}

\section{DISCUSSION}

We observed preterm MSAF in $4.3 \%$ of 1054 infants born at less than 33 weeks gestation. None of our 45 cases had Listeria infection. Although MSAF has been described in preterm infants with Listeria infection, ${ }^{56}$ they are generally ill in other ways at birth. Meconium stained liquor alone does not appear to be a useful indicator of Listeria infection. The three cases of Listeria infection observed in our institution during the study period had clear liquor. Other series of infants with preterm $\mathrm{MSAF}^{1-4}$ have not reported this outcome.

Mazor et al ${ }^{3}$ observed MSAF in 5.7\% of 4872 infants delivered at 24-37 weeks gestation. As in our study, this did not appear to be gestation related, as 5.6\% of infants of 3237 weeks gestation had MSAF. In a smaller study, Scott et al ${ }^{4}$ observed MSAF in $4.8 \%$ of infants delivered before 37 weeks. We chose to focus on more immature infants to enable us to study a population at greater risk of adverse perinatal outcome.

MSAF is a clinical diagnosis with no practicable confirmatory test. It is possible that in some cases in which MSAF was diagnosed, there may have been an alternative explanation for the discoloured liquor. None of the infants in our series had anatomical or functional intestinal obstruction diagnosed after birth. Some observers describe meconium as old, new, thick, thin, or particulate. These cannot be reliably defined and were not consistently recorded in this retrospective study. All MSAF was considered equivalent.

More of the mothers with MSAF in our study had prolonged rupture of the membranes. This was not associated with more histological chorioamnionitis or more positive cultures from the mothers. Mazor et $a^{3}$ also noted more premature rupture of the membranes in their series. In an earlier series where amniocentesis was performed on mothers in preterm labour, Mazor et al ${ }^{1}$ obtained more positive cultures from MSAF than from clear amniotic fluid. More of the infants with MSAF in our study received antibiotics for longer than 48 hours, but there were no more positive blood cultures. The cultures were performed on admission to the neonatal unit before the infants were started on antibiotics. The increased antibiotic use in the babies may reflect anxiety about the possibility of Listeria infection. The lack of positive

Table 4 Adverse neonatal outcomes

\begin{tabular}{llll}
\hline & Cases & Controls & Odds ratio $(95 \% \mathrm{Cl})$ \\
\hline Number & 45 & 41 & \\
Death & $10(22)$ & $4(10)$ & $2.64(0.76$ to 9.21$)$ \\
CLD (36 weeks) & $8(18)$ & $4(10)$ & $2.0(0.55$ to 7.22$)$ \\
PVL & $1(2)$ & $1(2)$ & $0.91(0.06$ to 15.02$)$ \\
IVH grade 3/4 & $5(11)$ & $0(0)$ & $2.03(1.62$ to 2.53$)$ \\
\hline
\end{tabular}

Data are number (\%).

CLD, Chronic lung disease; PVL, periventricular leucomalacia; IVH, intraventricular haemorrhage. 
cultures may be attributable, in part, to the maternal antibiotic exposure before delivery.

Concerns about the cardiotocographic traces were more common in mothers with MSAF in our study and in that of Mazor et al. ${ }^{3}$ Because of the difficulty of defining cardiotocographic abnormality in preterm infants and relating this to clinical outcome, we did not attempt to analyse this further. It is possible that the presence of meconium in the liquor made clinicians more likely to be concerned about the cardiotocographic traces. The infants with preterm MSAF were not more depressed at birth or more acidotic than controls in our study. Scott et $a l^{4}$ also found no significant difference in cord $\mathrm{pH}$ between infants with preterm MSAF and controls. We found no difference in first pH after birth.

More of our case infants were intubated at birth than controls. This difference was not significant and may be explained by our policy to suction directly the trachea in all infants with MSAF who are not vigorous at birth. No infant was diagnosed as having meconium aspiration syndrome. Although more infants with MSAF were intubated at birth, more control infants were treated with surfactant $(p=0.09)$. We used rescue surfactant treatment in intubated infants with clinical respiratory distress syndrome until 1999. From then on, prophylactic treatment was given to infants less than 29 weeks gestation and rescue treatment to more mature infants.

Although the affected infants were not in a worse condition at birth, preterm MSAF may reflect an earlier stressful event or illness, as some clinical outcomes appear to be worse. We observed significantly more severe intraventricular haemorrhages in infants with preterm MSAF than in controls. More infants died or developed chronic lung disease, although these differences were not significant.
Because of the low incidence of preterm MSAF, a very large study, gathering data from several centres, would be required to obtain statistical confidence on mortality data. Spinillo et $a l^{2}$ observed proportionately more cerebral palsy at 2 years of age among 17 preterm infants with MSAF than among 345 preterm infants with clear liquor.

In conclusion, preterm MSAF is observed in about $5 \%$ of preterm deliveries regardless of gestation. It is not a useful marker of intrapartum hypoxia or Listeria infection. However, it may identify a population of infants who will develop severe intraventricular haemorrhage or other adverse neurological outcome.

\section{Authors' affiliations}

A T Tybulewicz, Royal Hospital for Sick Children, Edinburgh, Scotland, UK

S K Clegg, B J Stenson, Neonatal Unit, Simpson Centre for Reproductive Health, Royal Infirmary, Little France, Edinburgh, Scotland, UK

G J Fonfé, St James's University Hospital, Leeds, UK

\section{REFERENCES}

1 Mazor M, Furman B, Wiznitzer A, et al. Maternal and perinatal outcome of patients with preterm labour and meconium-stained amniotic fluid. Obstet Gynecol 1995:86:830-3.

2 Spinillo A, Fazzi E, Capuzzo E, et al. Meconium stained amniotic fluid and risk for cerebral palsy in preterm infants. Obstet Gynecol 1997;90:519-23.

3 Mazor M, Hershkowitz R, Bashiri A, et al. Meconium stained amniotic fluid in preterm delivery is an independent risk factor for perinatal complications. Eur J Obstet Gynecol Reprod Biol 1998;81:9-13.

4 Scott H, Walker M, Gruslin A. Significance of meconium-stained amniotic fluid in the preterm population. J Perinatol 2001;21:174-7.

5 Halliday HL, Hirata T. Perinatal listeriosis: a review of twelve patients. Am J Obstet Gynecol 1979; 133:405-10.

6 Lennon D, Lewis B, Mantell C, et al. Epidemic perinatal listeriosis. Pediatr Infect Dis 1984;3:30-4. 